\title{
Number of Days from Date of Initial Pathologic Diagnosis to Date of Tumor Progression after Initial Treatment
}

National Cancer Institute

\section{Source}

National Cancer Institute. Number of Days from Date of Initial Pathologic Diagnosis to

Date of Tumor Progression after Initial Treatment. NCI Thesaurus. Code C158637.

The number of days from date of the initial cancer diagnosis to the date of tumor progression after the initial treatment. 